\title{
Investigating the holographic complexity in Einsteinian cubic gravity
}

\author{
Jie Jiang ${ }^{2, a}$, Banglin Deng ${ }^{1, b}$ \\ ${ }^{1}$ Department of Applied Physics, College of Geophysics, Chengdu University of Technology, Chengdu 610059, Sichuan, China \\ ${ }^{2}$ Department of Physics, Beijing Normal University, Beijing 100875, China
}

Received: 31 July 2019 / Accepted: 21 September 2019 / Published online: 10 October 2019

(C) The Author(s) 2019

\begin{abstract}
In this paper, we investigate the holographic complexity of a small mass AdS black hole in Einsteinian cubic gravity by using the "complexity equals action" (CA) and "complexity equals volume" $(\mathrm{CV})$ conjectures. In the CA context, the late-time growth rate satisfies the Lloyd bound for the $k=0$ and $k=1$ cases but it violates it for the $k=-1$ case in the first-order approximation of the small mass parameter. However, by a full-time analysis, we find that this latetime limit is approached from above, which implies that this bound in all of these cases will be violated. In the CV context, we considered both the original and the generalized CV conjectures. Differing from the CA conjecture, the late-time rate here is non-vanishing in the zeroth-order approximation, and this shows that the Lloyd bound is exactly violated even in the late-time limit. These results show numerous differences from the neutral case of the Einstein gravity in both the CA and the $\mathrm{CV}$ holographic contexts where all of their late-time results saturate the Lloyd bound. These differences illustrate the influence of the higher curvature correction in Einstein gravity.
\end{abstract}

\section{Introduction}

In recent years, the topic of "quantum complexity" has attracted growing interest in holography. There has been a growing interest in which complexity is defined as the minimum number of gates required to obtain a target state starting from a reference state [1,2]. From the holographic viewpoint, Brown et al. suggested that the quantum complexity of the state in the boundary theory corresponds to some bulk gravitational quantities which are called "holographic complexity". Then the two conjectures, "complexity equals volume" (CV) $[1,3]$ and "complexity equals action" (CA) $[4,5]$, were

\footnotetext{
a e-mail: jiejiang@mail.bnu.edu.cn

b e-mail: bldeng@aliyun.com
}

proposed. The CV conjecture states that the quantum complexity of the boundary state is given by the maximal volume of the bulk surface anchored by the time slices on the boundary, i.e.,

$C_{V}\left(\left|\psi\left(t_{L}, t_{R}\right)\right\rangle\right)=\frac{\max [V]}{\ell}$.

The CA conjecture states that the complexity of the boundary state is given by

$C_{A}\left(\left|\psi\left(t_{L}, t_{R}\right)\right\rangle\right) \equiv \frac{I_{\mathrm{WDW}}}{\pi \hbar}$,

where $I_{\mathrm{WDW}}$ is the on-shell action in the corresponding Wheeler-DeWitt (WDW) patch, which is enclosed by the past and future light sheets sent into the bulk spacetime from the time slices on the boundary. These conjectures have attracted many researchers to investigating the properties of both holographic complexity and circuit complexity in quantum field theory, e.g., [6-51]. As argued in [4], there is a bound on the complexity growth rate at late times,

$\dot{C} \leq \frac{2 M}{\pi \hbar}$,

which may be thought of as the Lloyd bound of the black hole system [52]. However, some further developments have cast the exact prefactor of this bound into question. In [15], the authors show that it is only applicable to quantum circuits made out of orthogonalizing gates. In the holographic context, this bound can only hold for a small black hole near a Hawking-Page phase transition [15]. Although it is not a precise feature of the complexity, we can also utilize it to illustrate characterizing the complexity in the different cases.

While the properties of the complexity in Einstein gravity have been investigated in many situations for both CA and $\mathrm{CV}$ conjectures, relatively little is known about its behavior in higher curvature gravitational theories, especially its time-dependent behaviors. The holographic principle suggests that the higher-order corrections of the bulk action are 
dual to finite $N$ and finite coupling effects in the boundary CFT. To study the holographic complexity for a higher curvature gravity could help us to investigate the hidden structures caused by the higher-order correction. Therefore, it is necessary for us to study the features of the holographic complexity in higher curvature gravity and show the influences of the higher curvature correction.

One of the most suitable higher curvature gravitational theories for holographic applications is the 4-dimensional Einsteinian cubic gravity, which provides a holographic toy model of a non-supersymmetric CFT in three dimensions. In [16], the authors have given a general formula for the full action for general $F\left(R_{a b c d}\right)$ gravity and applied it to the calculation of the CA complexity in Einsteinian cubic gravity for a massless black hole solution. Their result shows that the late-time CA complexity growth rate, in this case, vanishes because the mass of the black hole also vanishes. To gain a better understanding of the holographic complexity in Einsteinian cubic gravity, in this paper, we consider a small mass AdS black hole solution and then study the time-dependent behaviors of the holographic complexity growth rate, and we take the Lloyd bound as the criterion to present the influence of the higher curvature term.

The structure of this paper is as follows. In Sect. 2, we review the critical Einsteinian cubic gravity and its small mass AdS solution. In Sect. 3, we apply CA conjecture to calculate the complexity growth rate of the small mass AdS black hole. In Sect. 3, we also study the holographic complexity by using the original $\mathrm{CV}$ conjecture and the generalized CV conjecture which is modified by the Wald entropy density. Finally, we conclude the paper in Sect. 5.

\section{Critical Einsteinian cubic gravity}

We start with a quick review of the 4-dimensional Einsteinian cubic gravity and its small mass AdS solution. As shown in [53], the corresponding bulk action of this theory is given by

$$
\begin{aligned}
I_{\text {bulk }} & =\int_{\mathcal{M}} d^{4} x \sqrt{-g} \mathcal{L} \\
& =\int_{\mathcal{M}} d^{4} x \sqrt{-g}(R-2 \Lambda+\lambda \mathcal{P}),
\end{aligned}
$$

where the cubic invariant polynomial term $\mathcal{P}$ of the Riemann tensor is

$$
\begin{aligned}
\mathcal{P}= & 12 R_{a c b d} R^{c e d f} R_{e}{ }^{a} f^{b}+R_{a b}{ }^{c d} R_{c d}{ }^{e f} R_{e f}{ }^{a b} \\
& -12 R_{a b c d} R^{a c} R^{b d}+8 R_{a}{ }^{b} R_{b}{ }^{c} R^{a}{ }_{c}
\end{aligned}
$$

and $\lambda$ is a coupling constant. The equation of motion can be written as

$$
\psi_{a c d e} R_{b}^{c d e}-\frac{1}{2} g_{a b} \mathcal{L}-2 \nabla^{c} \nabla^{d} \psi_{a c d b}=0
$$

where we denote

$$
\begin{aligned}
\psi_{a b c d}= & \frac{\partial \mathcal{L}}{\partial R^{a b c d}} \\
= & \frac{1}{2}\left(g_{a c} g_{b d}-g_{a d} g_{b c}\right)+6 \lambda\left(R_{a d} R_{b c}\right. \\
& -R_{a c} R_{b d}+g_{b d} R_{a}^{e} R_{c e}-g_{a d} R_{b}{ }^{e} R_{c e} . \\
& -g_{b c} R_{a}^{e} R_{d e}+g_{a c} R_{b}{ }^{e} R_{d e}-g_{b d} R^{e f} R_{a e c f} \\
& +g_{b c} R^{e f} R_{a e d f}+g_{a d} R^{e f} R_{b e c f}-g_{a c} R^{e f} R_{b e d f} \\
& \left.-3 R_{a}{ }^{e} d^{f} R_{b e c f}+3 R_{a}{ }^{e}{ }^{f} R_{b e d f}+\frac{1}{2} R_{a b}{ }^{e f} R_{c d e f}\right) .
\end{aligned}
$$

The critical relation of this theory can be written as

$\Lambda=-\frac{2}{L^{2}}, \quad \lambda=-\frac{L^{4}}{24}$.

As mentioned in the introduction, in order to discuss the behaviors of the CA conjecture in Einsteinian cubic gravity, such as the Lloyd bound, here we consider a static small mass AdS black hole solution [54], in which the mass term can be regarded as a small perturbation of the solution. The line element can be written

$d s^{2}=-f(r) d t^{2}+\frac{d r^{2}}{f(r)}+r^{2} d \Omega_{2, k}^{2}$,

where

$$
f(r)=\frac{1}{L^{2}}\left(r^{2}-r_{h}^{2}\right)[1-m \tilde{f}(r)]
$$

is the blackening factor with

$$
\begin{aligned}
\tilde{f}(r) & =\frac{r^{2}+r_{h} r+2 r_{h}^{2}}{2 \mu r_{h}^{2}\left(r+r_{h}\right)}-\frac{r^{2}-r_{h}^{2}}{4 \mu r_{h}^{3}} \ln \left|\frac{r+r_{h}}{r-r_{h}}\right|, \\
r_{h}^{2} & =L^{2}(\mu-k),
\end{aligned}
$$

in the first leading order of the mass parameter $m$. Here $k=1,0,-1$ denotes the 2-dimensional spherical, planar, and hyperbolic geometry, respectively, $L$ is the AdS radius, and $\mu$ is a dimensionless parameter. This solution describes an asymptotic AdS black hole with a horizon at $r=r_{h}$. According to the blackening factor, we can see that this geometry has a curvature singular at the horizon under the firstorder approximation of $m$. However, this black hole is regular at the zeroth-order approximation. Therefore, we hope that the small mass correction does not affect the singularity at the horizon. This can be understood if the blackening factor is a nonanalytic function of the mass parameter, which means that when all corrections are concerned, this singularity will disappear.

According to the discussion in [54], under the first-order perturbation, the mass of this black hole can be expressed as

$M=4 \Omega_{2, k} m$. 


\section{CA conjecture}

In this section, we evaluate the CA complexity growth rate of the small mass AdS black hole. As suggested by [10], the CA complexity is equal to the full on-shell action on the WDW patch, which includes not only the bulk action but the surface terms, corner terms and counter term as well. According to [16], for the Einsteinian cubic gravity, the total action is given by

$$
\begin{aligned}
I= & I_{\text {bulk }}+4 \sum_{s}\left(\int_{\mathcal{B}_{s}} d^{3} x \sqrt{|h|} \Psi^{a b} K_{a b}\right) \\
& +(-1)^{\lambda} \int_{\mathcal{C}_{\lambda}} d^{2} x \sqrt{\sigma} \hat{\Psi} \eta_{\lambda} \\
& -\int_{\mathcal{N}} d \lambda d^{2} x \sqrt{\sigma} \hat{\Theta} \ln \left(l_{\mathrm{ct}} \Theta\right),
\end{aligned}
$$

where $\hat{\Theta}=\frac{1}{\sqrt{\sigma}} \partial_{\lambda}(\hat{\Psi} \sqrt{\sigma})$ and $\Theta=\frac{1}{\sqrt{\sigma}} \partial_{\lambda} \sqrt{\sigma}$ is the expansion scalar of the null generator with $l_{c t}$ an arbitrary length scale. The auxiliary fields can be written as

$$
\begin{aligned}
\Psi_{a b} & =\psi_{a c b d} n^{c} n^{d}, \\
\hat{\Psi} & =\psi^{a b c d} \epsilon_{a b} \epsilon_{c d},
\end{aligned}
$$

in which $n^{a}$ is the outward-directed normal vector of the corresponding surface, and $\epsilon_{a b}$ is the binormal of the joints.

Next, we evaluate the change of the action growth rate on the WDW patch. As illustrated in the Penrose diagram of this AdS spacetime in Fig. $1, I\left(t_{L}, t_{R}\right)$, denoting the action for the WDW patch determined by the time slice on the left and right $\mathrm{AdS}$ boundaries, is invariant under the time translation $I\left(t_{L}+\delta t, t_{R}-\delta t\right)=I\left(t_{L}, t_{R}\right)$. Thus the action growth can

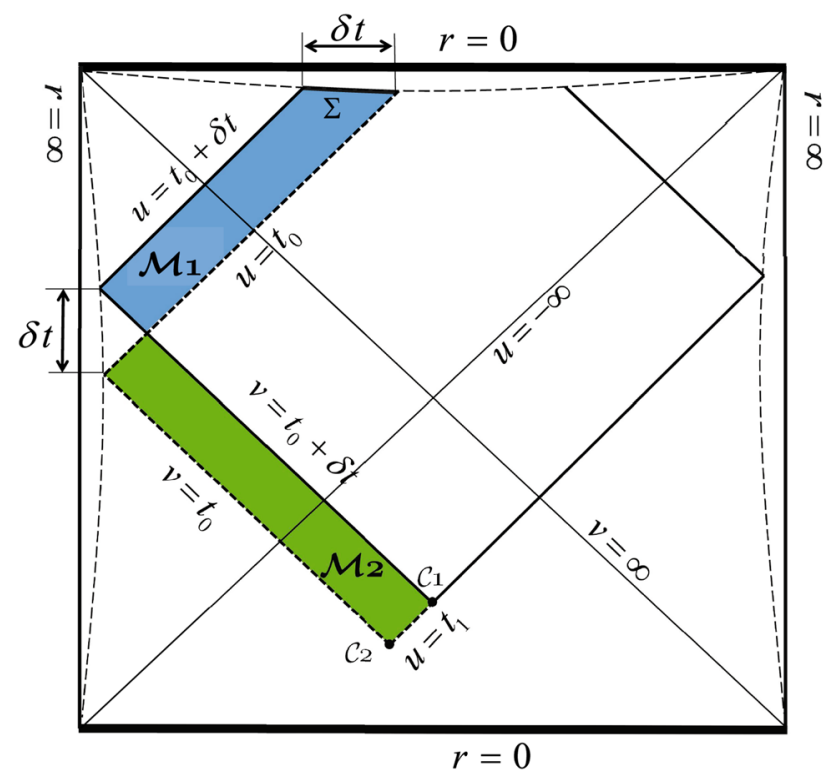

Fig. 1 Wheeler-DeWitt patches of a small mass AdS black hole in Einsteinian cubic gravity be computed as $\delta I=I\left(t_{0}+\delta t, t_{1}\right)-I\left(t_{0}, t_{1}\right)$, where the time on the right boundary has been fixed. To regulate the divergence near the AdS boundary, a cut-off surface $r=r_{\Lambda}$ is introduced. In addition, we also introduce a spacelike surface $r=\epsilon$ to avoid running into the spacelike singularity inside of the black hole. In addition, for simplicity we shall adopt the affine parameter for the null generator of null segments such that the surface term vanishes for all null segments. With all this in mind, we have

$\delta I=I_{\delta \mathcal{M}}+I_{\Sigma}+I_{\delta \mathcal{C}}+\delta I_{\mathrm{ct}}$,

with $I_{\delta \mathcal{M}}=I_{\mathcal{M}_{1}}-I_{\mathcal{M}_{2}}$ and $I_{\delta \mathcal{C}}=I_{\mathcal{C}_{1}}-I_{\mathcal{C}_{2}}$. Here $\mathcal{M}_{1}$ is bounded by $u=t_{0}, u=t_{0}+\delta t, v=t_{0}+\delta t$, and $r=r_{\text {min }}$. $\mathcal{M}_{2}$ is bounded by $u=t_{0}, v=t_{0}, v=v_{0}+\delta t$, and $u=t_{1}$. The null coordinates are defined as $u=t+r^{*}(r)$ and $v=$ $t-r^{*}(r)$ with

$$
\begin{aligned}
r^{*}(r)= & -\int_{r}^{\infty} \frac{d r}{f(r)} . \\
\simeq & \frac{L^{2}}{2 r_{h}} \ln \left|\frac{r-r_{h}}{r+r_{h}}\right|+\frac{m L^{2}}{4 r_{h}^{3}\left(r+r_{h}\right) \mu} \\
& \times\left[2 r_{h}^{2}+\left(r+r_{h}\right)^{2} \ln \left|\frac{r-r_{h}}{r+r_{h}}\right|\right]
\end{aligned}
$$

to the first leading order of $m$. Then, by using these coordinates, the typical point $r_{1}$ can be obtained by

$r^{*}\left(r_{1}\right)=-\frac{t}{2}$.

With the above preparation, we first calculate the change of the action which is contributed by the bulk region. Through straightforward calculation, we have

$$
\begin{aligned}
I_{\delta \mathcal{M}}= & \Omega_{2, k} \delta t \int_{0}^{r_{1}} d r r^{2} L \\
\simeq & 4 \Omega_{2, k} r_{1} \delta t\left(\mu-\frac{r_{1}^{2}}{L^{2}}\right)-\frac{m \Omega_{2, k} r_{1} \delta t}{L^{2} \mu} \\
& \times\left[4 r_{h}+2 r_{1}+\frac{4 k L^{2} r_{1}}{r_{h}^{2}}-\frac{6 r_{1}^{3}}{r_{h}^{2}}\right. \\
& \left.+\left(2 k L^{2}+3 r_{h}^{2}-3 r_{1}^{2}\right) \frac{r_{h}^{2}-r_{1}^{2}}{r_{h}^{3}} \ln \left(\frac{r_{h}-r_{1}}{r_{1}+r_{h}}\right)\right],
\end{aligned}
$$

where we used the limit process $\epsilon \rightarrow 0$. Then we proceed to the surface contribution from the spacelike surface $r=\epsilon$. By using (13) and (9), we have

$$
\begin{aligned}
I_{\Sigma} & =4 \int_{\Sigma} d^{2} x \sqrt{-h} \Psi^{a b} K_{a b} \\
& \simeq 4 m .
\end{aligned}
$$

To evaluate the corner contributions from $\mathcal{C}_{1}$ and $\mathcal{C}_{2}$, we first choose $k_{1 a}=\nabla_{a} u$ and $k_{2 a}=-\nabla_{a} v$ as the null generator of the past right and past left null boundaries separately. By 
using these expressions, we obtain $k_{1} \cdot k_{2}=-2 / f$ and

$$
\begin{aligned}
\hat{\Psi}= & -4\left(1-\frac{\mu L^{2}}{r^{2}}\right) \\
& -\frac{m}{\mu}\left[\frac{4 r_{h}}{r^{2}}-\frac{\left(r^{2}-r_{h}^{2}\right)}{r_{h}^{3} r^{2}} \ln \left|\frac{r+r_{h}}{r-r_{h}}\right|\right. \\
& \left.\times\left(3 r_{h}^{2}-r^{2}+2 k L^{2}\right)-\frac{2}{r_{h}^{2} r}\left(r^{2}-r_{h}^{2}-2 k L^{2}\right)\right] .
\end{aligned}
$$

Then, by using (13) and the transformation parameter $\eta_{\lambda}=$ $\ln \left(k_{1} \cdot k_{2} / 2\right)$, one can further obtain

$$
\begin{aligned}
I_{\delta \mathcal{C}}= & \left.\Omega_{k, 2} \delta r \frac{\partial}{\partial r}[\Phi(r) \ln (-f(r))]\right|_{r=r_{1}} \\
= & -\frac{\Omega_{k, 2} \delta t}{2} \\
& \times\left[\Phi\left(r_{1}\right) f^{\prime}\left(r_{1}\right)+\Phi^{\prime}\left(r_{1}\right) f\left(r_{1}\right) \ln \left(-f\left(r_{1}\right)\right)\right]
\end{aligned}
$$

where we have used

$\delta r=r_{1}-r_{2}=-\frac{1}{2} f\left(r_{1}\right) \delta t$

and we denoted

$\Phi(r)=r^{2} \hat{\Psi}(r)$.

Finally, we consider the counter term contributions. By the translation symmetry, there are only two null segments contributing to the action growth. The first one comes from the null segment $u=t_{1}$ with $r$ as the affine parameter, i.e., $k_{1}^{a}=\left(\frac{\partial}{\partial r}\right)^{a}$, which gives rise to the expansion $\Theta=2 / r$. Then the counter term of the past right null segment can be written

$I_{\mathrm{ct}}^{(1)}=-\Omega_{2, k} \int_{r_{1}}^{r_{\Lambda}} d r \Phi^{\prime}(r) \ln \left(\frac{d l_{\mathrm{ct}}}{r}\right)$.

Then we have

$\delta I_{\mathrm{ct}}^{(1)}=-\frac{\Omega_{2, k} \delta t}{2} \Phi^{\prime}\left(r_{1}\right) f\left(r_{1}\right) \ln \left(\frac{d l_{\mathrm{ct}}}{r_{1}}\right)$.

With similar calculation, we can easily find $I_{\mathrm{ct}}^{(2)}=I_{\mathrm{ct}}^{(1)}$. By summing all the previous results, one can obtain

$$
\begin{aligned}
\frac{d C_{A}}{d t}= & \frac{M}{\pi \hbar}\left[1+\frac{r_{1}^{4}}{r_{h}^{2} L^{2} \mu}+\frac{r_{1}^{3}\left(r_{h}^{2}-r_{1}^{2}\right)}{2 r_{h}^{3} L^{2} \mu} \ln \left(\frac{r_{1}+r_{h}}{r_{h}-r_{1}}\right)\right. \\
& \left.-\frac{1}{8 m} \Phi^{\prime}\left(r_{1}\right) f\left(r_{1}\right) \ln \left(-\frac{4 l_{\mathrm{ct}}^{2} f\left(r_{1}\right)}{r_{1}^{2}}\right)\right] .
\end{aligned}
$$

At the late times, we have $r_{1} \rightarrow r_{h}$. Then the complexity growth rate becomes

$$
\lim _{t \rightarrow \infty} \frac{d C_{A}}{d t}=\left(2-\frac{k}{\mu}\right) \frac{M}{\pi \hbar} .
$$

We can see that, for the planar case $(k=0)$, the late-time CA complexity growth rate saturates the Lloyd bound, for

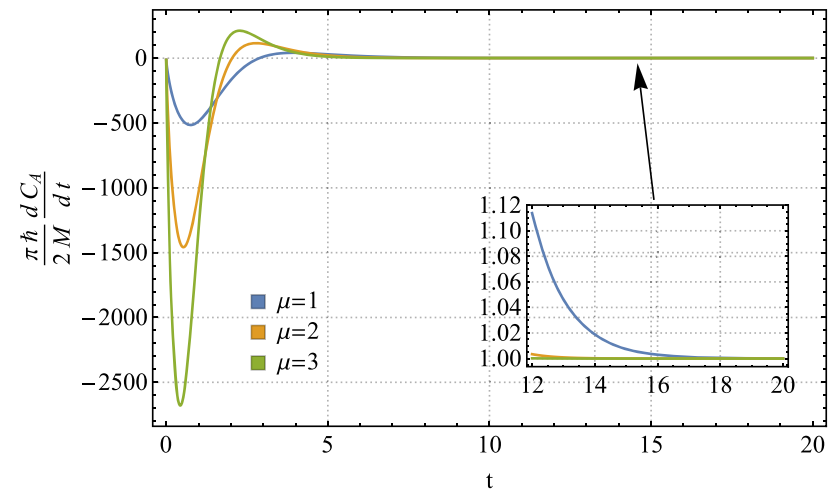

Fig. 2 The CA complexity growth rate for the planer case, where we set $k=0, l_{\mathrm{ct}}=1, L=1$ and $m=0.01$

spherical case $(k=1)$, it is lower than the saturated value, while for the hyperbolic case $(k=-1)$, the late-time result will violate this bound. These results give different features with the neutral case of Einstein gravity, where the late-time limit in all of these cases saturate the Lloyd bound.

Finally, we consider the time dependence of the CA complexity growth rate. From (16) and (17), one can obtain

$$
\begin{aligned}
r_{1}= & r_{h} \tanh \left(\frac{r_{h} t}{2 L^{2}}\right)+\frac{m}{\mu} \\
& \times \frac{L^{2}\left(1+e^{r_{h} t / L^{2}}\right)^{2}-2 r_{h} t e^{2 r_{h} t / L^{2}}}{L^{2}\left(1+e^{r_{h} t / L^{2}}\right)^{3}} .
\end{aligned}
$$

By using this expression, we show the time dependence of the CA complexity growth rate in Fig. 2. In this figure, we can see that the late-time limit is approached above, which implies that the Lloyd bound will also be violated when we consider the full-time evolution of the complexity. Moreover, we can also find that the maximal value of the complexity increase with the value of parameter $\mu$ (Fig. 2).

\section{$4 \mathrm{CV}$ conjecture}

In this section, we consider the $\mathrm{CV}$ conjecture and apply it into the calculation of the complexity growth rate. According to (3), evaluation of the $\mathrm{CV}$ complexity boils down to finding the maximal surface of the volume function,

$V(\Sigma)=\frac{1}{\ell} \int_{\Sigma} d^{3} x \sqrt{|h|}$

with some length scale $\ell$. However, it is also expected [35] that in the presence of higher derivative terms one needs to extend the notion of volume to a new quantity. The situation is similar to thermal and entanglement entropies where the area must be replaced by a certain functional to be evaluated on the horizon and RT curve, respectively. Motivated by the Wald formula, one can consider a generalized volume which 


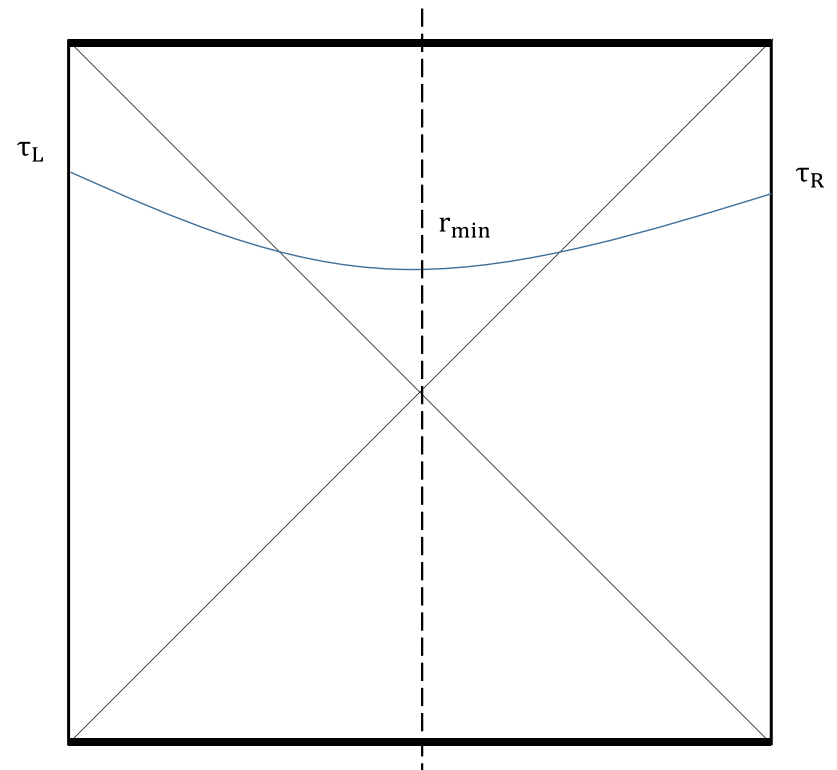

Fig. 3 "Complexity equals volume" (CV) duality for the eternal AdS black hole dual to the boundary state. The blue curve denotes a spacelike surface which has maximal volume (generalized volume)

is modified by a Wald-like form, i.e., the maximal surface is obtained by maximizing the generalized volume function

$V_{g}(\Sigma)=\frac{1}{\ell} \int_{\Sigma} d^{3} x \hat{\Psi} \sqrt{|h|}$,

and then the holographic complexity is the generalized volume of its maximal surface. Next, we turn to evaluating the holographic complexity growth rate by using these two conjectures. For convenience, here we denote the volume function

$V(\Sigma)=\int_{\Sigma} d^{3} x \sqrt{h} W$

where $W=1$ for the original CV conjecture and $W=\hat{\Psi} / \ell$ for the generalize $\mathrm{CV}$ conjecture. Then we need to obtain the maximal surface which is bounded by the two spacelike slices at the boundary time $t_{L}$ on the left side and $t_{R}$ on the right side (Fig. 3). Following the discussion in [3], we use the Eddington-Finkelstein coordinates. Then the line element becomes

$d s^{2}=-f(r) d u^{2}+2 d u d r+r^{2} d \Omega_{2, k}^{2}$.

By considering the symmetries of this spacetime, this codimension one surface $\Sigma$ can be thought of as the direct product of a 2 -sphere and a curve in the $u-r$ plane, i.e.,

$\Sigma=\gamma \times \Sigma_{k}^{2}$

$\gamma \mapsto(u(\lambda), r(\lambda))$, where the curve $\gamma$ is given in parametric form. Therefore, the generalized volume is given by

$V_{g}[\gamma]=\Omega_{2, k} \int_{\lambda_{0}}^{\lambda_{f}} d \lambda r^{2} \sqrt{-f(r) \dot{u}^{2}+2 \dot{u} \dot{r}} W(r)$.

The largest surface $\Sigma$ is determined by the curve $\gamma$ that maximizes this integral, which is the same as solving for the equation of motion with the Lagrangian

$\mathcal{L}_{V}=r^{2} \sqrt{-f(r) \dot{u}^{2}+2 \dot{u} \dot{r}} W(r)$.

Since $\mathcal{L}_{V}$ does not depend explicitly on $u$, we can find the conserved quantity

$E=-\frac{\partial \mathcal{L}_{V}}{\partial \dot{u}}=\frac{r^{2}(f \dot{u}-\dot{r}) W(r)}{\sqrt{-f \dot{u}^{2}+2 \dot{u} \dot{r}}}$.

Due to the reparametrization invariance of our final result, for simplification, we choose $\lambda$ as the proper generalized volume as follows:

$r^{2} \sqrt{-f(r) \dot{u}^{2}+2 \dot{u} \dot{r}} W(r)=1$.

Using (36) and (37), we can further obtain

$E=r^{4}(f \dot{u}-\dot{r}) W^{2}$,

$r^{8} W^{4} \dot{r}^{2}=E^{2}+r^{4} f W^{2}$.

Using the shift symmetry, we can always choose $t_{R}=-t_{L}=$ $t / 2$. Then this surface can be composed of two equivalent parts. So, we can only restrict ourselves to the range $t>0$. For the turning point $r=r_{\min }$, we have $\dot{r}=0$ and $t_{\min }=0$, which means

$E=-r_{\min }^{2} W\left(r_{\min }\right) \sqrt{-f\left(r_{\min }\right)}$.

The maximal generalized volume can be written as

$V_{\text {gen }}=2 \Omega_{2, k} \int_{r_{\text {min }}}^{\infty} d r \frac{r^{4} W^{2}}{\sqrt{E^{2}+r^{4} W^{2} f}}$.

Therefore, to obtain this holographic complexity, we first need to obtain the relationship between the boundary time $t$ and the radius $r_{\min }$ of the turning point. From (38), we can further obtain

$\frac{t}{2}-u_{\min }=\int_{r_{\min }}^{\infty} d r\left(\frac{E}{f \sqrt{E^{2}+r^{4} W^{2} f}}+\frac{1}{f}\right)$.

By the fact $t_{\min }=0$, i.e., $u_{\min }=r_{\min }^{*}$, we can obtain

$t=2 \int_{r_{\min }}^{\infty} d r \frac{E}{f \sqrt{E^{2}+r^{4} W^{2} f}}$,

which means that the time $t$ can be regarded as a function of $r_{\text {min }}$. Together with (40) and (42), the CA complexity growth rate can be further obtained,

$\frac{d C_{V}}{d t}=\frac{\Omega_{2, k}}{\ell} \sqrt{-f\left(r_{\min }\right)} r_{\min }^{2} W\left(r_{\min }\right)$. 


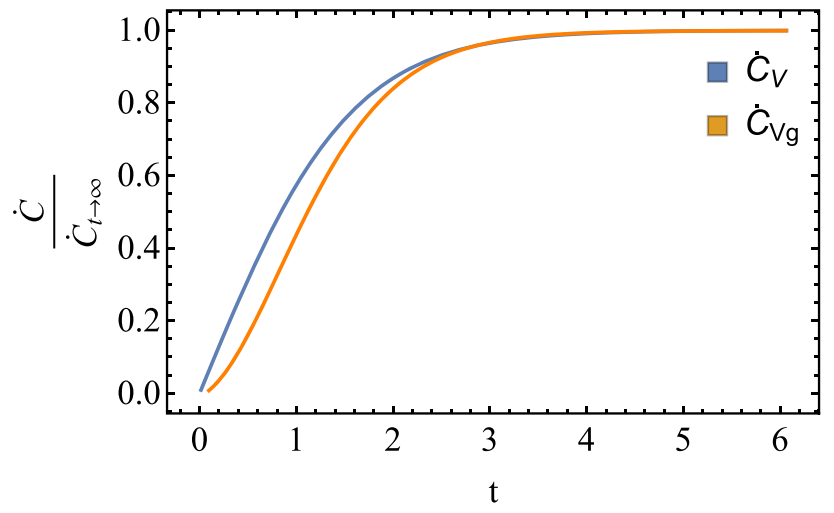

Fig. 4 The CV complexity growth rate for the planar case for both the $\mathrm{CV}$ conjecture and the generalized $\mathrm{CV}$ conjecture, where we set $k=0, l_{\mathrm{ct}}=1, L=1$ and $m=0.01$

Using this relation, we can obtain the full-time dependence of this holographic complexity. One can see that this complexity explicitly depends on the function $W$.

At late times, since the turning radius $r_{\text {min }}$ decreases when the boundary time increases, together with (38) and (39), the minimal possible value of $r_{\min }$ should be the extreme value point $\tilde{r}_{\text {min }}$ of the function $\sqrt{-f\left(r_{\min }\right)} r_{\min }^{d-1} W\left(r_{\min }\right)$. Then we have

$\lim _{t \rightarrow \infty} \frac{d C_{V}}{d t}=\frac{\Omega_{2, k}}{\ell} \sqrt{-f\left(\tilde{r}_{\min }\right)} \tilde{r}_{\min }^{2} W\left(\tilde{r}_{\min }\right)$.

With these preparations, next, we turn to considering these two special CV conjectures: the original $\mathrm{CV}$ conjecture and generalized $\mathrm{CV}$ conjecture which is modified by the Wald entropy density.

For the original $\mathrm{CV}$ conjecture, by setting $W=1$ and using the line element (10), we can further obtain the latetime result of the complexity growth rate

$\lim _{t \rightarrow \infty} \frac{d C_{V}}{d t} \simeq \frac{\Omega_{2, k}}{L \ell}\left(\frac{2 r_{h}^{3}}{3 \sqrt{3}}-0.22 \frac{m r_{h}^{2}}{\mu}\right)$.

By a similar calculation, the late-time limit of the generalized CV complexity rate is given by

$\lim _{t \rightarrow \infty} \frac{d C_{\mathrm{Vg}}}{d t} \simeq \frac{4 L \mu r_{h}}{\ell}-\frac{2 m L}{\mu \ell}\left(2 \mu-\frac{2 r_{h}^{2}}{L^{2}}\right)$.

From these results, we can find that the late-time growth rate in these conjectures have non-vanishing zeroth-order contributions, which implies that the CV complexity has a non-zero growth rate even for the massless solution, and the Lloyd bound must be violated in these cases even though we only consider the late-time limit, which is obviously different from the result of the Schwarzschild-AdS black hole for Einstein gravity. These differences must be caused by the higher curvature correction of the gravitational theory.
Finally, we show the time dependence of the holographic complexity for these two CV conjectures in Fig. 4 for the planar case $(k=0)$. From this figure, we can see that both of the $\mathrm{CV}$ holographic complexities increase with time and finally saturate the late-time value, which shows similar behaviors to the $\mathrm{CV}$ conjecture in the neutral case for Einstein gravity.

\section{Conclusion}

According to the calculation for Einsteinian cubic gravity in [16], the authors found that the late-time CA complexity growth rate of the massless black hole vanishes. Their result is in agreement with the Lloyd bound since the mass of this black hole also vanishes. In this paper, in order to gain a better understanding of the holographic complexity in Einsteinian cubic gravity, we study the holographic complexity growth rate of a small mass AdS black hole with both $\mathrm{CA}$ and $\mathrm{CV}$ conjectures. In the CA context, we find that the late-time growth rate only contains the first-order contributions of $\delta m$. Moreover, different from the neutral case of the Einstein gravity, where the late-time growth rate of complexity saturates the bound, here the late-time result shows other features. For the planar case $(k=0)$, it saturates the Lloyd bound, for the spherical case $(k=1)$, the late-time value is lower than the saturated value of this bound, while for the hyperbolic case $(k=-1)$, it will violate the bound. By showing the time dependence of the growth rate, we find that the late-time value is approached, i.e., the complexity has a non-zero growth rate even for the massless case, which might imply that the Lloyd bound will also be violated after the time evolution of the complexity is taken into account. In the $\mathrm{CV}$ context, we considered both the original and the generalized CV conjectures. Different from the CA results, its late-time limit growth rate has non-vanishing zeroth-order contributions of $m$, which means that the Lloyd bound will be violated even though we only consider the late-time limit. The above results show numerous differences from the neutral case of the Einstein gravity in both the CA and CA contexts where all of their late-time results saturate the Lloyd bound. These illustrate the influence of the higher curvature correction in the Einstein gravity.

Acknowledgements This research was supported by National Natural Science Foundation of China (NSFC) with Grants No. 11675015, the Cultivating Program of Excellent Innovation Team of Chengdu University of Technology (Grant No. KYTD201704), the Cultivating Program of Middle-aged Backbone Teachers of Chengdu University of Technology (Grant No. 10912-2019KYGG01511), the Open Research Fund of Computational Physics Key Laboratory of Sichuan Province, Yibin University (Grant No. JSWL2018KFZ01).

Data Availability Statement This manuscript has no associated data or the data will not be deposited. [Authors' comment: Data sharing 
not applicable to this article as no datasets were generated or analysed during the current study.]

Open Access This article is distributed under the terms of the Creative Commons Attribution 4.0 International License (http://creativecomm ons.org/licenses/by/4.0/), which permits unrestricted use, distribution, and reproduction in any medium, provided you give appropriate credit to the original author(s) and the source, provide a link to the Creative Commons license, and indicate if changes were made.

Funded by SCOAP ${ }^{3}$.

\section{References}

1. L. Susskind, Fortsch. Phys. 64, 24 (2016)

2. S. Aaronson, arXiv: 1607.05256

3. D. Stanford, L. Susskind, Phys. Rev. D 90, 126007 (2014)

4. A.R. Brown, D.A. Roberts, L. Susskind, B. Swingle, Y. Zhao, Phys. Rev. Lett. 116, 191301 (2016)

5. A.R. Brown, D.A. Roberts, L. Susskind, B. Swingle, Y. Zhao, Phys. Rev. D 93, 086006 (2016)

6. D.A. Roberts, D. Stanford, L. Susskind, Localized shocks. JHEP 1503, 051 (2015). https://doi.org/10.1007/JHEP0(2015)051

7. L. Susskind, Y. Zhao, arXiv:1408.2823

8. J. Jiang, Phys. Rev. D 98, 086018 (2018)

9. R.G. Cai, S.M. Ruan, S.J. Wang, R.Q. Yang, R.H. Peng, Action growth for AdS black holes. JHEP 1609, 161 (2016). https://doi. org/10.1007/JHEP09(2016)161

10. L. Lehner, R.C. Myers, E. Poisson, R.D. Sorkin, Phys. Rev. D 94, 084046 (2016)

11. R.A. Jefferson, R.C. Myers, JHEP 1710, 107 (2017)

12. K. Hashimoto, N. Iizuka, S. Sugishita, Phys. Rev. D 96, 126001 (2017)

13. D. Carmi, S. Chapman, H. Marrochio, R.C. Myers, S. Sugishita, JHEP 1711, 188 (2017)

14. J. Jiang, J. Shan, J. Yang, arXiv:1810.00537

15. W. Cottrell, M. Montero, Complexity is simple!. JHEP 1802, 039 (2018)

16. J. Jiang, H. Zhang, Phys. Rev. D 99, 086005 (2019)

17. J. Jiang, M. Zhang, arXiv: 1905.07576

18. M. Guo, J. Hernandez, R.C. Myers, S.M. Ruan, arXiv:1807.07677

19. Z.Y. Fan, M. Guo, arXiv: 1811.01473

20. Z.Y. Fan, M. Guo, JHEP 1808, 031 (2018)
21. R.Q. Yang, Y.S. An, C. Niu, C.Y. Zhang, K.Y. Kim, arXiv: 1809.06678

22. Y.S. An, R.G. Cai, Y. Peng, arXiv:1805.07775

23. R.Q. Yang, Y.S. An, C. Niu, C.Y. Zhang, K.Y. Kim, arXiv:1803.01797

24. Y.S. An, R.H. Peng, Phys. Rev. D 97, 066022 (2018)

25. A. Reynolds, S.F. Ross, Class. Quant. Grav. 34, 175013 (2017)

26. S. Chapman, H. Marrochio, R.C. Myers, JHEP 1701, 062 (2017)

27. J. Jiang, X. Liu, Phys. Rev. D 99, 026011 (2019)

28. J. Jiang, Eur. Phys. J. C 79, 130 (2019)

29. J. Jiang, X.W. Li, arXiv:1903.05476

30. J. Jiang, B.X. Ge, Phys. Rev. D 99, 126006 (2019)

31. X.H. Feng, H.S. Liu, arXiv: 1811.03303

32. J. Jiang, B. Deng, X.W. Li, arXiv: 1908.06565

33. D. Carmi, R.C. Myers, P. Rath, JHEP 1703, 118 (2017)

34. B. Czech, Phys. Rev. Lett. 120, 031601 (2018)

35. P. Caputa, N. Kundu, M. Miyaji, T. Takayanagi, K. Watanabe, JHEP 1711, 097 (2017)

36. M. Alishahiha, Phys. Rev. D 92, 126009 (2015)

37. P. Caputa, N. Kundu, M. Miyaji, T. Takayanagi, K. Watanabe, Phys. Rev. Lett. 119, 071602 (2017)

38. A.R. Brown, L. Susskind, Phys. Rev. D 97, 086015 (2018)

39. C.A. Agon, M. Headrick, B. Swingle, arXiv:1804.01561

40. O. Ben-Ami, D. Carmi, JHEP 1611, 129 (2016)

41. S. Chapman, M.P. Heller, H. Marrochio, F. Pastawski, Phys. Rev. Lett. 120, 121602 (2018)

42. Y. Zhao, Phys. Rev. D 97, 126007 (2018)

43. Z.Y. Fan, H.Z. Liang, arXiv: 1908.09310

44. Z.Y. Fan, M. Guo, Phys. Rev. D 100, 026016 (2019)

45. Z. Fu, A. Maloney, D. Marolf, H. Maxfield, Z. Wang, JHEP 02, $072(2018)$

46. L. Hackl, R.C. Myers, arXiv:1803.10638

47. M. Alishahiha, A. Faraji Astaneh, M.R. Mohammadi Mozaffar, A. Mollabashi, arXiv:1802.06740

48. J. Couch, S. Eccles, W. Fischler, M.L. Xiao, JHEP 1803, 108 (2018)

49. B. Swingle, Y. Wang, arXiv: 1712.09826

50. M. Moosa, JHEP 1803, 031 (2018)

51. B. Chen, W.M. Li, R.Q. Yang, C.Y. Zhang, S.J. Zhang, arXiv: 1803.06680

52. S. Lloyd, Nature 406, 1047 (2000)

53. P. Bueno, P.A. Cano, Phys. Rev. D 94, 104005 (2016)

54. X.H. Feng, H. Huang, Z.F. Mai, H. Lu, Phys. Rev. D 96, 104034 (2017) 